\title{
Modelling complex for surface radar images in the "distance-angle" coordinates
}

\author{
Yury Markov*, Alexander Bokov, and Vladimir Vazhenin \\ Ural Federal University, Yekaterinburg, Russian Federation
}

\begin{abstract}
The mathematical modelling software complex of monostatic radar systems is discussed in the article. It is aimed to model of typical radar scenarios with various conditions of an electromagnetic waves reflection from terrain. The received signals are used to synthesize radar images (portraits) of surfaces. The structure of the modelling complex allows studying of single-channel and multi-channel synthetic aperture radars with pulse and continuous types of modulation of signals reflected from underlying surfaces of different types. The complex can be useful to simulate radar portraits of the surrounding radar scene's objects in the native "distance-angle" coordinates, including the nadir direction of the antenna system. The portraits for cases of front-side looking by a widepattern antenna in two adjacent directions are modelled and evaluated in the article.
\end{abstract}

\section{Introduction}

Non-contact methods of obtaining information about the Earth's surface, objects on it or in its bowels constitute the basis of Earth remote sensing [1-3]. In the typical case of active airborne and space radar systems, the quantitative characteristics of the electromagnetic field radiated and reflected by the terrain (power, propagation time, spectral composition, phase, polarization properties, etc.) are evaluated to collect sufficient information about the illuminated and observed radar scene [3-5]. Special signal processing and radar image computation is performed on board or at a ground station using the digitized signal data, which have undergone preliminary processing and transmitted from the radar's carriers.

Due to the complexity and costs of natural simulation, it is necessary to use mathematical modelling of the radar scene and signal processing to develop and verify the operation of such radar systems [6-8].

The specific task considered in the paper is the study and refinement of the technology for modelling signals, and radar images of the underlying surface based on them in the native "distance-angle" coordinates. The signals of airborne radar systems are simulated, including those with multi-channel radar systems (multi-channel antenna and receivers), which operate with the possibility of using them both for measuring motion parameters, observing the terrain, and for integration with other navigation and monitoring systems.

*Corresponding author: asdal@mail.ru 


\section{The structure of the modelling complex}

The mathematical modelling software for modelling multi-channel radar systems can be divided into two organizational levels: a single-channel version and multi-channel version of the modelling complex. The structure of the single-channel version of the software complex is shown in Fig. 1.

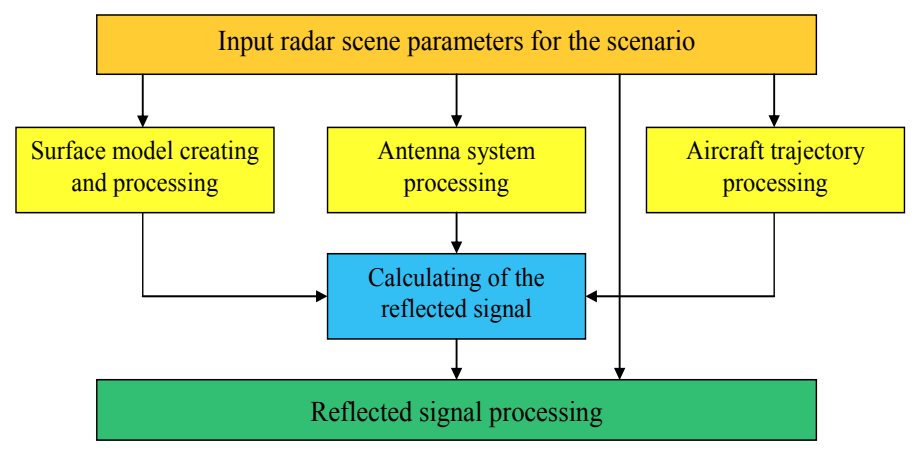

Fig. 1. The structure of the single-channel version of the modeling complex.

At the first stage of modelling, it is necessary to enter initial data for modelling various components of the radar scene and scenario. Some initial data may be common for different elements of the general model. Based on the input data entered, a model of a reflecting surface, a model of an antenna system, and a model of an aircraft flight trajectory are built simultaneously or sequentially. The models of the antenna system and aircraft trajectory take into account the gain coefficients of the receiving and transmitting antennas, their antenna patterns, aircraft angle evolutions. Various conditions of electromagnetic wave propagation and reflection from surface reflective elements should be taken into account by the terrain model [7-10]. It is not discussed here due to its complexity.

When building a signal model for the case of multi-channel radars, the single-channel version is used modularly. The general block diagram of the multi-channel version of the complex for modelling signals and their processing is presented in Fig. 2.

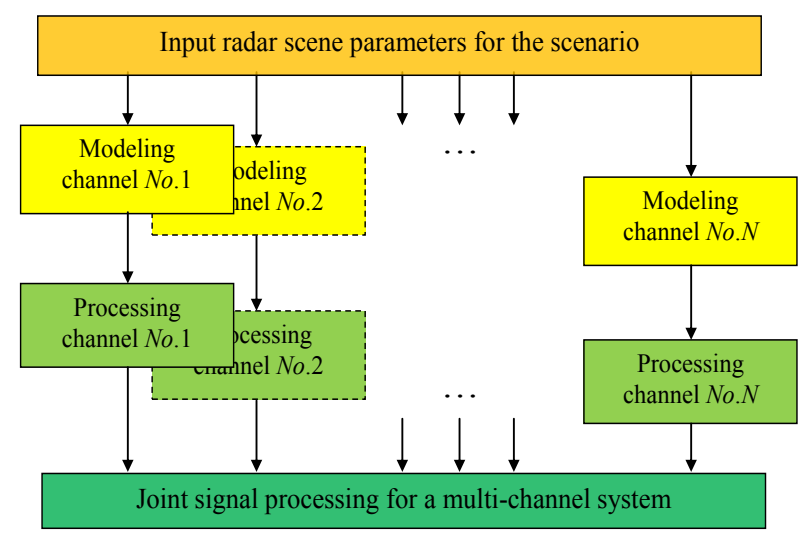

Fig. 2. The general structure of the multi-channel version of the modelling complex.

When modeling a multi-channel system, it is assumed that each of the modeled channels is independent. That is, each of the channels is a signal received by one receiving antenna. In this case, the transmitting antenna can be either common for all or a group of channels, or its own for each $[2,5]$. 


\section{Some simulation results}

\subsection{Simulation conditions}

A number of model experiments were carried out, signals were modelled, and radar images were synthesized for the C-band radar systems with pulse and continuous radiation for horizontal motion of a radar platform above a flat rough uniform surface. The geometry of the antenna system for multi-channel radar (in this case, two-channel) is shown in Fig. 3. Note here, that in our following simulations, a width of an antenna pattern of $50^{\circ}$ was used. This is a peculiarity of our research, because a typical synthetic aperture radar (SAR) do not use such a wide antenna pattern.

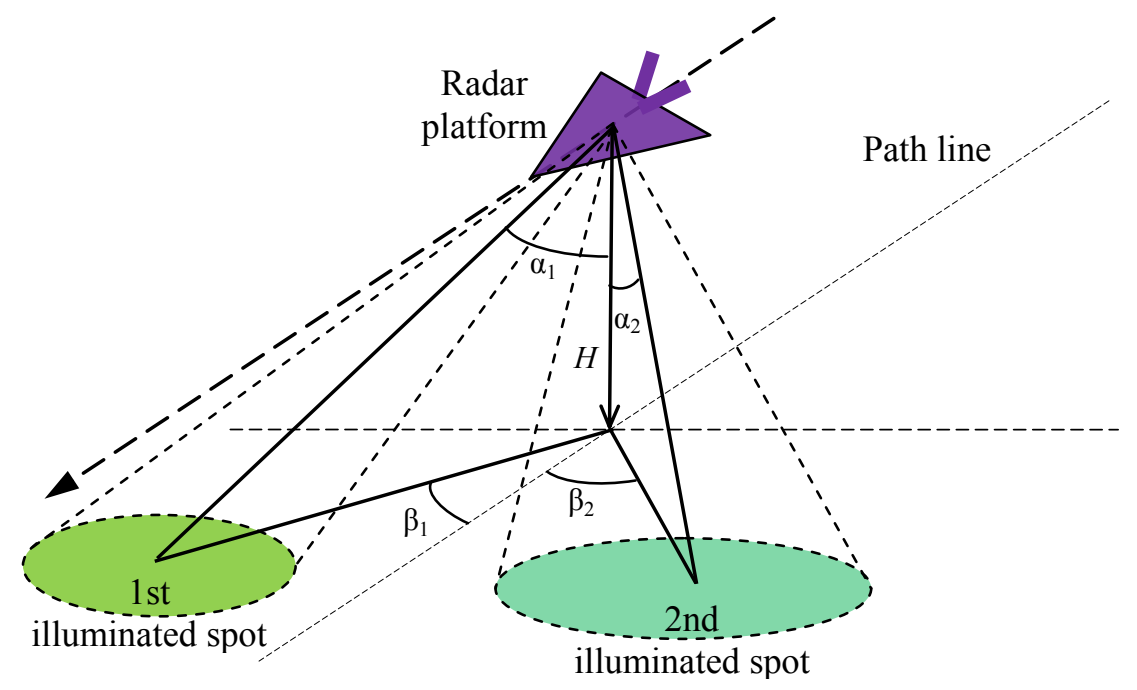

Fig. 3. The geometry of the radar scene and the antenna system.

In Fig. 3 both angles $\alpha$ is the antenna deviation of the vertical axe in the vertical plane, both angles $\beta$ is the antenna deviation of the path line in the horizontal plane, $H$ is the radar platform altitude. For a remote sensing radar with continuous radiation, a frequency deviation of $150 \mathrm{MHz}$ was chosen, therefore, the range resolution is $1 \mathrm{~m}$. For radar with pulsed radiation, the duration of the probe pulse was chosen to be $10 \mathrm{~ns}$, and, accordingly, the range resolution is $3.33 \mathrm{~m}$. Also, in our following experiments, the flight altitude is 100 or $200 \mathrm{~m}$, the horizontal speed of the the radar platform is $100 \mathrm{~m} / \mathrm{s}$, the synthesis aperture (distance) is $5 \mathrm{~m}$. So, the correspinding synthesis time is $50 \mathrm{~ms}$.

\subsection{Single-channel radar images}

Using the discussed modeling software complex on the basis of the basic signal model and typical processing algorithm, a number of model experiments were conducted. Surface radar images were synthesized for various flight conditions and two types of sounding signals for a various number of channels.

First of all, radar images were synthesized for a radar of the centimeter-wavelength range (C-band) with vertical (nadir) radiation (thus, in Fig. 3, the angles $\alpha_{1}$ and $\beta_{1}$ are equal to $0^{\circ}$, and the second antenna is absent) over a flat rough uniform surface with a different number of reflecting elements on the surface and with two altitudes equal to 100 and $200 \mathrm{~m}$. 
The synthesized comparative radar images are shown in Fig. 4, 5. The horizontal axis on the obtained images shows the focus angle (in degrees), on the vertical axis is the distance to the focus area (in the elements of range resolution). The maximum viewing angle in all the graphs is $180^{\circ}$, despite the fact that the possible observation around the nadir irradiation is $360^{\circ}$. This is because the speeds and Doppler frequency shifts on the right and left of the aircraft path line for different reflectors is the same and indistinguishable for the radars of this single-channel type.

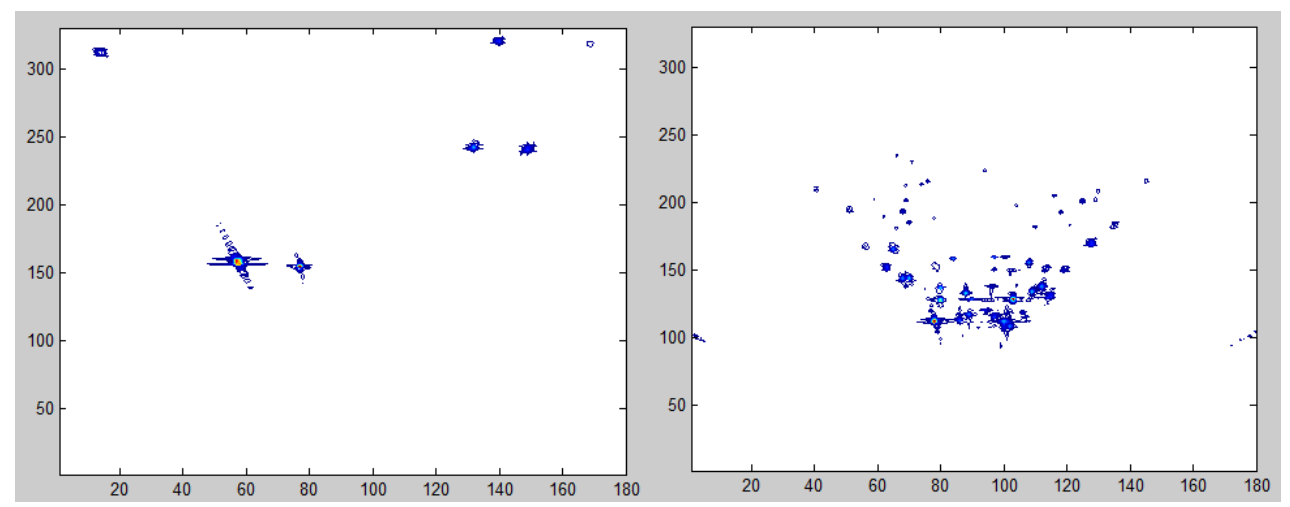

Fig. 4. Comparative images for continuous-waveform radiation with a different number of simulated reflectors: 10 on the left and 250 on the right; other conditions are the same: height is $100 \mathrm{~m}$, horizontal speed is $100 \mathrm{~m} / \mathrm{s}$.

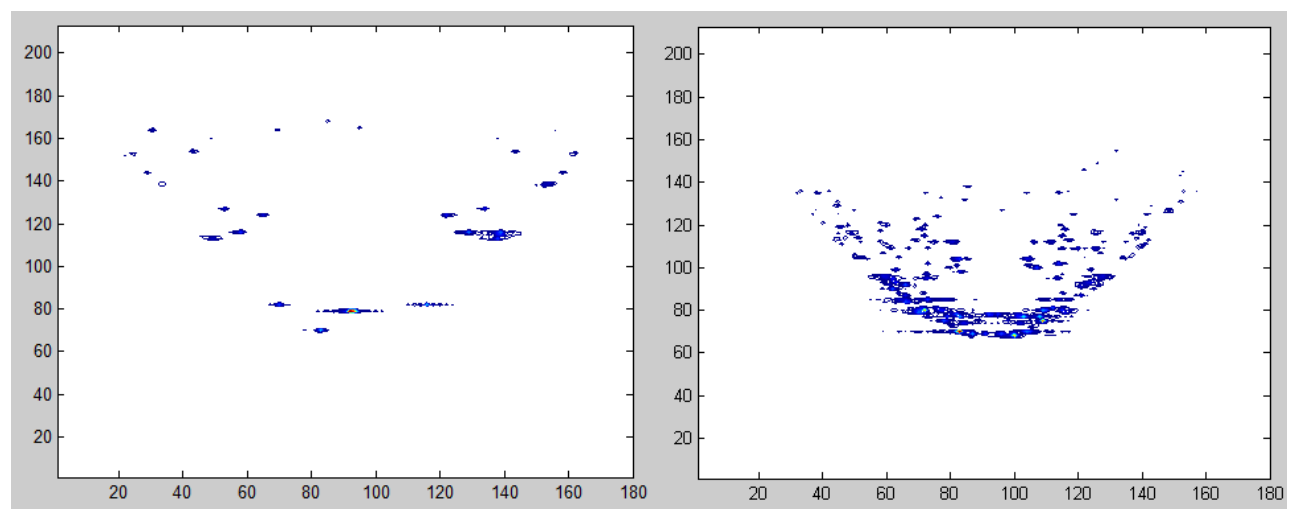

Fig. 5. Comparative images for pulse radiation with a different number of simulated reflectors: 50 on the left and 500 on the right; other conditions are the same: height is $200 \mathrm{~m}$, horizontal speed is $100 \mathrm{~m} / \mathrm{s}$.

Reflectors, which can represent reflective bright points or facets of modeled objects and surfaces, are located in the surface plane. They are clearly distinguishable on the presented radar images. Their number corresponds to the number of reflectors specified in the used signal model. For the distances shorter than the radar platform altitude all the images does not contain any marks (in the absence of a significant level of additive noise), since there are no reflecting objects at such slant ranges.

The largest mark amplitudes are registered from the reflectors with small slant ranges (close to vertical) and small angles is caused by the greater energy reflected by the subradar zone, due to the small attenuation of the signal at the backscattering pattern and antanna pattern peaks. With an increase in the distance from both the radar platform and from the subradar zone, the level of reflected signals decreases, which is clearly seen in threedimensional image presentation shown in Fig. 6. You can also see that according to the 
coordinates corresponding to large angles and small distances, there are no reflecting objects, because according to the rules of stereometry they would have to lie outside the plane of the underlying surface, and such objects were not specified when modeling the surface. Therefore, in general, the reflectorive area synthesized by radar imaging lies inside the hyperbola.

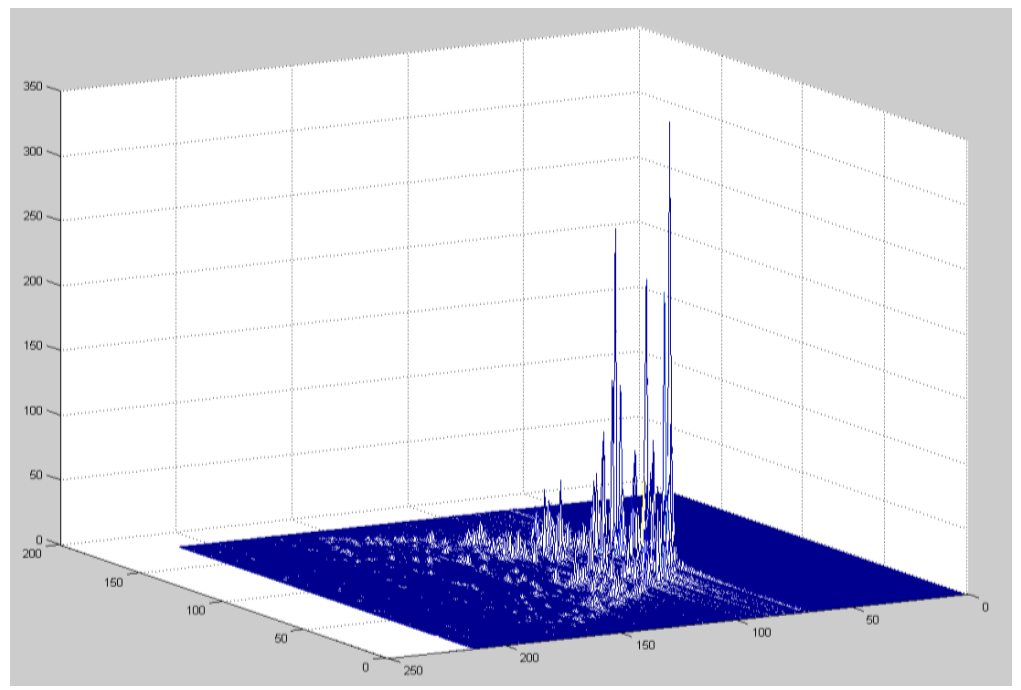

Fig. 6. Radar image of simulated surface: 3D-portrait in the coordinates "distance-angle".

SAR's images with continuous radiation are characterized by a cross-shaped shape of marks from objects (see Fig. 4), which is explained by the presence of side lobes both for the signal spectrum reflected from the point target and for convolution along the angular coordinate. For SAR's images with pulsed radiation, the reflector marks do not have side lobes along the distance coordinate and are simply elongated along the angular coordinate.

A basic observing radars traditionally obtain two-dimensional images of the Earth's surface in the native "distance-azimuth" coordinates. On the contrary, tipycal mapping SAR with side and front-side looking obtain radar images in the "longitudinal range - transverse range" coordinates [2-4], which is more suitable for human percepation. Unfortunatelly, the last require more processor resources to recounting, interpolation of the obtained data samples and geometric correction of images.

So, for relative navigation methods without human actions, the native coordinate system is simpler and cheaper to implement. In addition, radar images in the coordinates "distanceangle" is convenient from the point of view of matching the pixel pitch of the radar image with the resolution of the radar. It is suitable for detecting radio-contrast objects with dimensions corresponding to the actual resolution of the radar in accordance with the corresponding spatial coordinates.

\subsection{Two-channel radar images}

In the next experiment, radar images were synthesized for a two-channel C-band radar with front-side looking (see Fig. 3). When modelling in this experiment, it was assumed that all the angles $\alpha_{1,2}$ and $\beta_{1,2}$ are equal to $30^{\circ}$, the surface consists of 100 reflectors.

The synthesized radar images are shown in Fig. 7, 8. The horizontal axis on the obtained images shows the focus angle (in degrees), on the vertical axis is the distance to the focus area (in the elements of range resolution). Both comparative radar images were synthesized for the same surface using two-channel independent signal processing. 

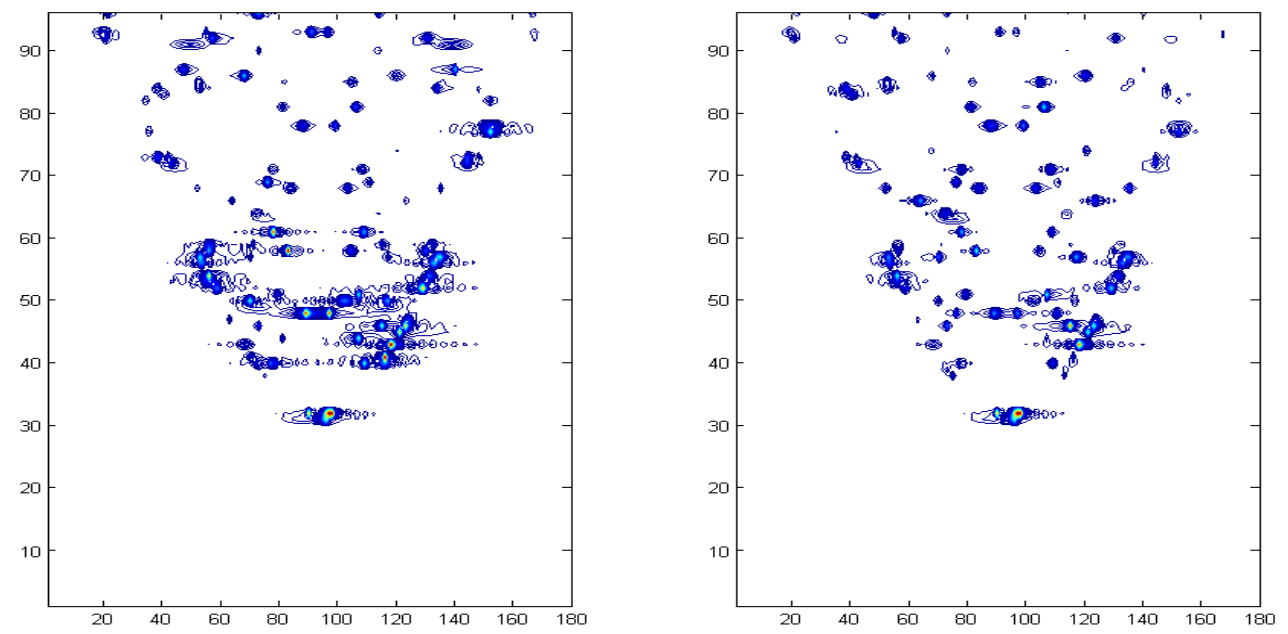

Fig. 7. Comparative radar images for two-channel radar with pulse radiation.
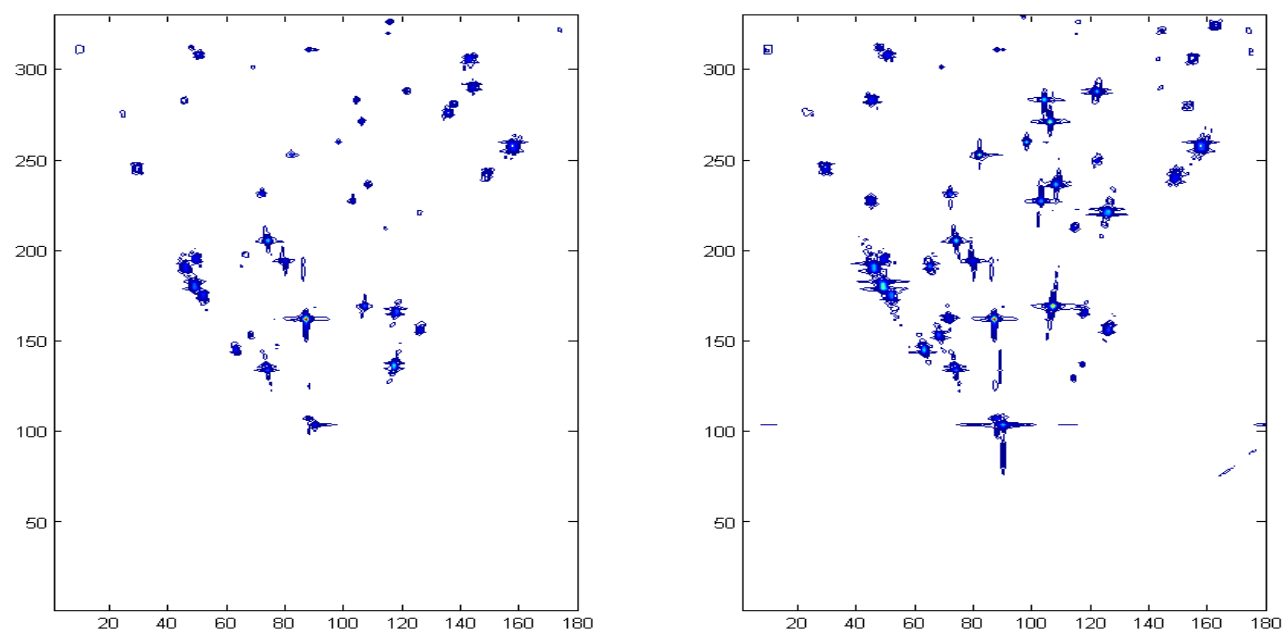

Fig. 8. Comparative images for two-channel radar with continuous-waveform radiation.

In the synthesized radar images, an incomplete correlation between the radar images for different channels is noticeable. This is due to the fact that both images display the same surface, illuminated differently by antennas. Some elements on the surface turned out to be more noticeable for one channel, some for another. This is due to the fact that the antennas are pointed in different directions and receive a signal from the surface in different ways. Reflections from surface/object reflectors (facets) are observed by different channels at different angles, and, due to non-isotropic antenna patterns, with different power levels of the corresponing marks.

Despite the fact that both pulsed and continuous-wave radars were simulated with observing of the same surface, the correlation between the radar images with different signal modulation is not clear. This is caused by a large number of reflectors, which complicates the human perception.

In Fig. 9, 10 two comparative radar images are shown for the same conditions as above, but with the number of reflectors equal to 10 . 

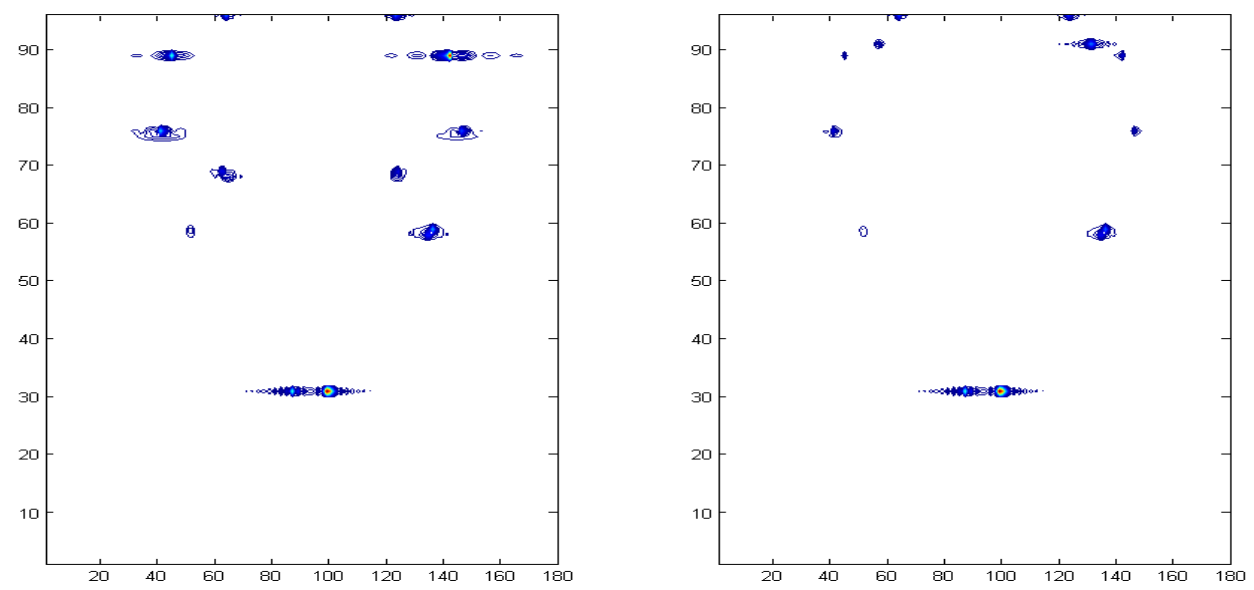

Fig. 9. Comparative images for two-channel radar with pulse radiation, only the 10 brightest reflectors are modelled.
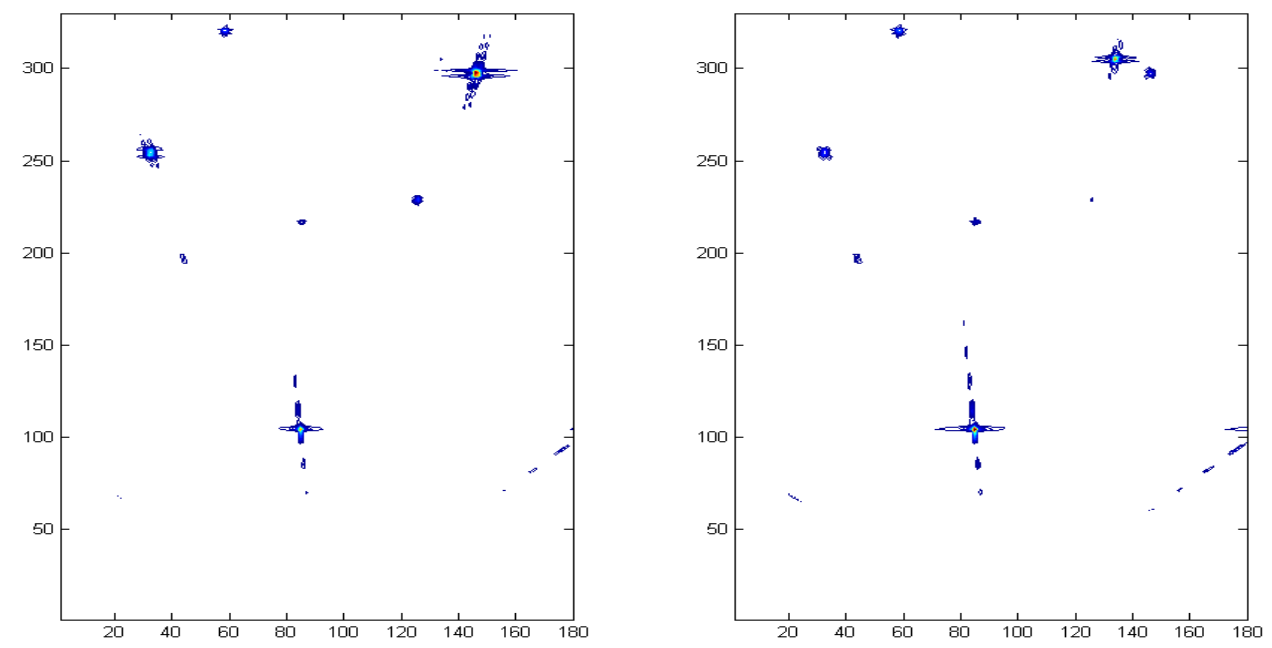

Fig. 10. Comparative images for two-channel radar with continuous-waveform radiation, only the 10 brightest reflectors are modelled.

When analyzing the given radar images, it should be taken into account that the scales along the vertical axis do not coincide. The axes are graduated in range resolution elements and the scales differ by 3.33 times.

In the latest model experiment, radar has similar characteristics, but with a different configuration of the antenna system. The angles $\alpha_{1,2}$ and $\beta_{1}$ are still $30^{\circ}$, and the angle $\beta_{2}$ is $90^{\circ}$ (see Fig. 3), the surface consists of 100 reflectors. The synthesized radar images are shown in Fig. 11, 12. The horizontal axis on the obtained images shows the focus angle (in degrees), and the vertical axis is the distance to the focus area (in the elements of range resolution). Both comparative radar images were synthesized for the same surface using two-channel independent signal processing.

There is a noticeable correlation between these synthesized radar images obtained for the directions of the first and second antennas. This similarity of images is due to the fact that both modelled radar antennas have a very wide antenna patterns. So, each of them receives a sum of signals reflected from the common parts of their illuminated spots. In addition, each image has especially emphasized reflective objects, which are especially 
intensely irradiated with the antenna of this antennas' direction (beam). And since the difference in the deviation angles between the "beams" in this experiment is greater than in the previous one, the degree of correlation is less.



Fig. 11. Comparative radar images for two-channel radar with pulse radiation.

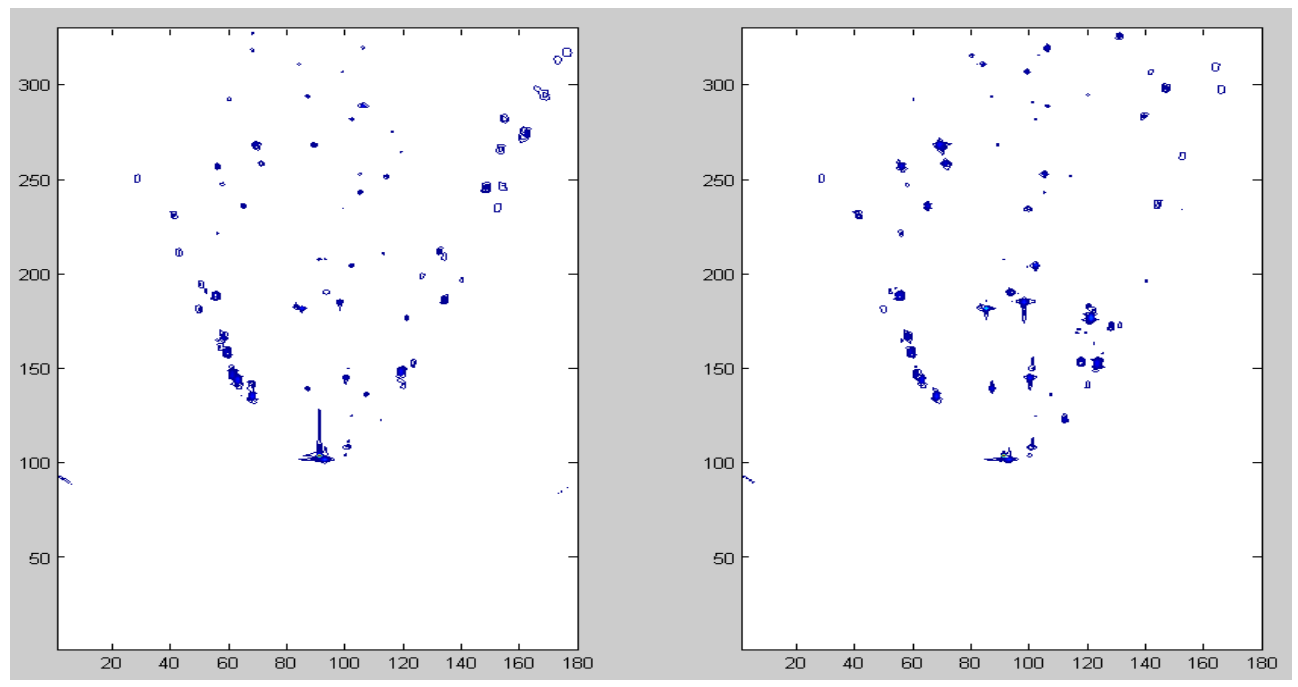

Fig. 12. Comparative images for two-channel radar with continuous-waveform radiation.

The similarity of images in the region near the nadir point is especially strong. In other words, objects located in the minimum slant ranges are illuminated by two antennas of a two-channel radar receiver, so this can be useful to provide additional safity and accuracy in solving the navigation problem, for example, by using the interferometric signal processing technique.

\section{Conclusion}

In this paper the multi-channel software complex for modelling signals and radar surface portraits in the native "distance-angle" coordinates is proposed. It is based on the developed mathematical model of reflected signals and typical SAR processing methods. It includes modelling of the about nadir direction of the main lobe of the antenna pattern. Moreover, 
the multi-channel radar images are taken for a single surface generation, which yields coherent signals and may be used in joint complex radar images processing. The radar images of the surface were obtained under various conditions and radar operation parameters, types of underlying surface. Their analysis was presented.

As a result, it is worth emphasizing that multi-channel radar systems with about nadir illumination can make a stepchange in micronavigation technique of various vehickles, because it can prvide both safe navigation and location of reflective objects from whole surrounding radar scene.

\section{Acknowledgments}

This research has been supported by the grant of the Ministry of Science and Higher Education of the Russian Federation (project No. 8.2538.2017/4.6).

\section{References}

1. A. I. Baskakov, T. S. Zhutyayeva, Yu. I. Lukashenko, Locational Methods for Studying Objects and Media (in Russian, Academy, Moscow, 2011)

2. G. S. Kondratenkov, A. U. Frolov, Radio vision. Radar Earth `s remote sensing systems (in Russian, Radiotechnica, Moscow, 2005)

3. M. I. Skolnik, Radar Handbook (3rd edn., The McGraw-Hill Companies, 2008)

4. V. G. Kobernichenko, Radioelectronic Earth's remote sensing systems (in Russian, UrFU, Yekaterinburg, 2016)

5. V.S. Verba, Detection of Ground Objects. Radar-based Airborne Detection and Guidance Systems (in Russian, Radio Engineering, Moscow, 2007)

6. B. R. Mahafza, Radar Systems Analysis and Design Using MATLAB (Chapman and Hall/CRC, 2013)

7. A. S. Bokov, A. K. Sorokin, A. E. Smertin, E. F. Zapolskikh, V. G. Vazhenin, CEURWS, 2076, 29-38 (2018)

8. E. F. Zapolskikh, A. E. Smertin, V. G. Vazhenin, A. S. Bokov, RSEMW-2017, 234237 (2017)

9. A. K. Fung, K. S. Chen, Microwave Scattering and Emission Models for Users (Artech house, USA, 2010)

10. F. T. Ulaby, M. C. Dobson, Handbook of radar scattering statistic for terrain (Artech house, USA, 1989) 